\title{
Methods to Perform Opinion Mining and Sentiment Analysis to Detect Factors Affecting Mental Health
}

\section{Ravindra Kumar}

\begin{abstract}
Sentimental analysis and opinion extraction are emerging fields at AI. These approaches help organizations to use the opinions, sentiments, and subjectivity of their consumers in decision-making. Sentiments, views, and opinions show the feeling of the consumers towards a given product or service. In recent years, Opinion Mining and Sentiment Analysis has become an important tool to detect the factors affecting mental health. It's Also true that human biasness is available in giving opinions, but it can be eliminated through the use of algorithms to get better results. However, it is crucial to remember that the developers are human and might pass the biasness to the algorithms during training. The main target of this paper is to give background knowledge on opinion extraction and sentimental analysis and how factors affecting mental health can be collected. The paper aimed to use interested individuals in knowing some of the algorithms in opinions extraction and sentimental analysis. The paper also provides benefits of using sentiment analysis and some of the challenges of using the algorithms.
\end{abstract}

Keywords: Opinion Mining and Sentiment Analysis has become an important tool to detect the factors affecting mental health.

\section{I.INTRODUCTION}

Sentiment analysis refers to the process of detecting positive or negative sentiment in a text. Most organizations have used sentiment analysis to improve their products and service provision. Through sentiment, analysis businesses detect sentiment in social data, which helps them determine the reputation of the brand and how to achieve customer satisfaction. Technological advancement has improved operations in all sectors. Social media sites are one of the platforms that organizations have ventured to help them in expanding their operations. Various organizations have rapidly adopted opinion mining and sentiment analysis (Vinodhini \& Chandrasekaran, 2012). The high usage of social media and the internet has allowed people to be more open about their feelings and thoughts than before. An excellent example of a product that became successful because of social media is vaping. When the first users of vaping devices posted on social media and expressed joy and comfort on the devices, more people were willing to try them.

Manuscript received on September 08, 2021.

Revised Manuscript received on September 15, 2021.

Manuscript published on October 30, 2021.

* Correspondence Author

Ravindra Kumar*, Technical Account Manager, Navvis Healthcare, St. Louis MO, 63021. Email: rakumar25@gmail.com

(c) The Authors. Published by Blue Eyes Intelligence Engineering and Sciences Publication (BEIESP). This is an open access article under the CC BY-NC-ND license (http://creativecommons.org/licenses/by-nc-nd/4.0/)
Sentiment analysis has become an essential tool needed by every organization to help in monitoring and understanding the opinions of their customers. Organizations can use opinions in survey responses and social media conversations to understand their customer feedback and tailor products to meet their needs. Emotions and thoughts make up human life, and one cannot visualize the future without addressing them. Opinion mining and sentiment analysis have been adopted as AI solutions to human biasness regarding opinions and sentiments on products and services.

\section{II.EXPERIMENT TO DETECT MENTAL HEALTH}

This experiment was conducted during EU S presidential elections for 2020 the participants were known and were able to provide feedback about their sentiments and opinions and feelings fairly there were there were two participants groups when group bird inclines towards Republicans and one group bars inclines towards Democrats to collect better data for this experiment we scrap the social media post of both groups we used Facebook Twitter and Instagram data to check on their analysis. Data was analyzed for following feelings.

- Is person feeling sad after reading social media post?

- Is person commenting on a social media post is confused by the behavior of his/her friend and it is resulting in reduced ability to concentrate?

- Is there a feeling of excessive fears, worries, or extreme guilt?

- Are users experiencing extreme mood changes?

- Are users feeling like withdrawing from friends and activities?

- Are there symptoms of significant tiredness, low energy or problems sleeping?

- Is there a feeling of delusions, paranoia or hallucination?

- Are users having difficultly to cope with daily problems or stress

- Are users taking more alcohol or using drugs to cope with social media?

- Are there major changes in eating habits?

- Is there a change in sex drive?

Same questions were send in a questionnaire, using google form, to both groups to get their opinion and matched with the result of sentiment analysis.

\section{III.TYPES OF SENTIMENT ANALYSIS}

The models of sentiment analysis are concerned with polarity, feelings and emotions, urgency, and intentions. Sentiment analysis has been classified at the documental levels, sentencing levels, and entity levels.

Published By: 
At the documental levels, the main concern is classifying opinions on a single subject. The main task at the document level avoid opinion making about any person or sentences in the essay. It is primarily used in supervised learning to classify documents (Singh \& Husain, 2014). Frequency numbers and single terms such as word portioning, inclusion of adjectives, and opinion expression, are also used in machine learning. The sentence level looks at each phrase, while the entity level looks at individual aspects in products.

A fine-grained sentiment analysis model is used by businesses that are interested in polarity. This type of sentiment analysis is vital in interpreting 5-star ratings in a review. The polarity components addressed here are (very positive, positive, neutral, negative, and very negative). The best products are rated under very positive, and the poorest are rated very negative.

Emotion detection is another type of sentiment analysis centered on emotions such as happiness, frustrations, anger, and sadness. The emotion detection systems use complex algorithms in machine learning or lexicons. It is, however, crucial to note that lexicons cannot be entirely relied upon because of the different word use by people (MonkeyLearn, n.d). Some might use words consider negative to portray how someone is making it in business. An example is "your customer base is fire you are killing it."

Customers also use text sentiments in product reviews of various products. In some instances, they might concentrate on the multiple aspects of development such as battery life or camera quality for mobile phones. Aspect-based sentiment analysis can allow organizations to understand the aspects that were rated positive, negative, or neutral. An aspect-based classifier helps in identifying and classifying the sentiments.

The main components of opinion mining include; opinion holder, opinion object, and opinion orientation. Opinions can be regular, comparative, explicit, and implicit (MonkeyLearn, n.d). Regular opinions can be guided or indirect. Examples of regular views include the image quality is HD. This is direct because the aspect addressed is image quality. Another example is on cream application I experience skin splitting. The unit being addressed is cream which is indirectly conveyed as a negative opinion.

Comparative opinions involve getting different opinions on the same unit, which contradicts normal opinions. An example is Samsung S6 has high processing than iPhone 6, but iPhone 6 metallic body is appealing than Samsung S6.

In explicit opinion, everything is defined clearly, such as this car is flashy. Implicit opinions, on the other hand, are implied but not stated clearly. Idioms and metaphors are also used in implicit opinions, which makes sentiment analysis complex.

\section{IV.ALGORITHMS AND TECHNIQUES IN OPINION EXTRACTION AND SENTIMENTAL ANALYSIS}

\section{A. Classification}

Classification is a technique used in opinion mining. Under classification, the case variety belong to particular classes and class attribute value.

\section{B. K-Nearest Neighbor Algorithm}

The algorithm is majorly applied in regressions and classifications together with the non-parametric procedure. Under this algorithm, the vectors having same class mark are shown in each training set existing in the multidimensional featured spaces.

\section{Support Vector Machine Algorithm}

The algorithm is a supervised classification of linear and non-linear form. SVM is mainly used to separate data sets. Under this algorithm, a line is picked up that divides two groups into two regions (Saraswati \& Tamilrasai, 2014). A high-order thinking is also used in mapping of non-linear data to linear.

\section{Rule-based Approach}

This approach is based on an algorithm that clearly defines the description of opinion to the entity. It dwells on identifying subjectivity, polarity, and the subject of opinion. The operations involved in this technique include; stemming, parsing, lexicon analysis, and tokenization (Singh \& Husain, 2014). Under this approach, there are two lists of words, one with positive and the other with negative. The algorithm then goes through the texts and identifies the words that match the categories. The overall results are then provident basing on the prominent category. Positive polarity is declared if most of the texts were found under the positives. The challenge with this approach is the lack of flexibility.

\section{E. Automatic Sentiment Analysis}

Automatic sentiment analysis is an upgrade of the rulebased approach. The technique uses machine learning to understand the meaning of each message. Due to this technique, it is more precise and accurate. Large textual materials can be analyzed without complications. It mainly involves the use of supervised machine learning classification algorithms.

\section{V.IMPORTANCE OF SENTIMENT ANALYSIS}

Through sentiment analysis, organizations use sentiment data to understand the perception of products in the market. Organizations can identify customer support correspondence regarding their product. It also helps in social media tractions and user-generated product reviews (Medhat et al. 2014). Sentiment analysis helps organizations customize their products to meet the feedback from their customers on social media platforms. Sentiment analysis allows organizations to gain market knowledge based on consumer standpoint. Companies can make future marketing strategies basing on the results from sentiment analysis. Marketing is improved because of the knowledge on the strengths and weaknesses of the product.

\section{VI.CHALLENGES OF SENTIMENT ANALYSIS}

Understanding human emotion is one of the challenging tasks in sentiment analysis. Context and polarity definition is one of the challenges in sentiment (Medhat et al. 2014).

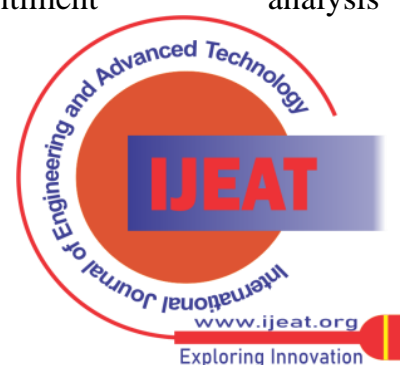


Humans can understand context easily compared to algorithms. Algorithms rely on human configuration on what to do, unlike humans, which can adjust based on situations. Hence additional tools for understanding the context of the message are used. Examples of such devices include word2vec and doc2vec.

\section{VII.CONCLUSION}

Opinion mine and sentimental analysis are the future tools in helping organizations and institutions in decision-making. Human bias has been eliminated in assessing opinions and emotions from consumers through the application of opinion extraction and sentimental analysis algorithms. A lot of effort needs to be done to eliminate the challenges faced by sentiment analysis algorithms.

\section{REFERENCES}

1. Medhat, W., Hassan, A., \& Korashy, H. (2014). Sentiment analysis algorithms and applications: A survey. Ain Shams engineering journal, 5(4), 1093-1113.

2. MonkeyLearn. (\.). Everything There Is to Know about Sentiment Analysis. Retrieved from https://monkeylearn.com/sentiment-analysis/

3. Saraswati, K., \& Tamilrasai, D. A. (2014). Investigation of SVM for Opinion Mining. Journal of theoretical and Apllied Information Technology, 59(2).

4. Singh, P. K., \& Husain, M. S. (2014). Methodological study of opinion mining and sentiment analysis techniques. International Journal on Soft Computing, 5(1), 11.

5. Vinodhini, G., \& Chandrasekaran, R. M. (2012). Sentiment analysis and opinion mining: a survey. International Journal, 2(6), 282-292.

\section{AUTHOR'S PROFILE}

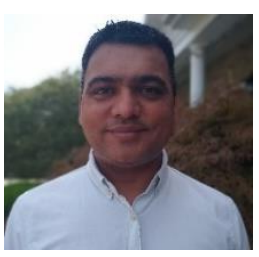

Ravindra Kumar, is a technologist in the healthcare IT, population health management, clinical software applications product management, and Implementation space. Ravindra Kumar is interested in social use of Artificial Intelligence and spending his time in researching how to find ways using AI to save human life and make human life better and easy. He has over fifteen years of proven experience in leading and making global teams spanning various functions like Technical, Solutions Architecture, Technical Alliances, Development, and Quality. He is passionate about value-based care and is actively involved in designing and implementing new approaches for data-driven initiatives for population health management for Medicare Advantage and ACO markets. In Past, Ravindra was involved in end to end implementation of Medicare Advantage plans, and Population health IT systems. He has good knowledge of care management, Utilization Management and other applications for value based care. He has a great interest in healthcare data analytics and is pretty comfortable in using Tableau, SQL, PowerBI for analysis of various healthcare data (Claims, eligibility, provider data, etc), including EMRs, HL7, ADT and FHIR records. He also loves to train people in quality practices, including RPA(Robotic Process Automation), Test Automation, and Agile engineering.

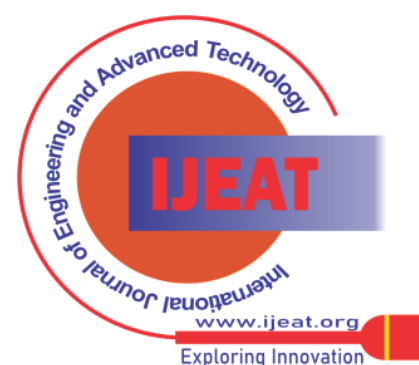

